\title{
Atuação do fisioterapeuta no NASF-AB: possibilidades e desafios
}

\author{
The role of physiotherapists in $\mathrm{CFH}-\mathrm{PC}$ : possibilities and challenges
}

\author{
Sabrina Oliveira Viana ${ }^{1}$ \\ Orcid: https://orcid.org/0000-0002-7031-596X \\ Maria Clara Botelho Vieira Amorim ${ }^{3}$ \\ Orcid: https://orcid.org/0000-0002-3544-3157
}

Dayane Jhenifer Ribeiro Silva ${ }^{2}$

Orcid: https://orcid.org/0000-0002-2744-2461

\begin{abstract}
Resumo
Introdução: O Fisioterapeuta está presente em todos os NASF-AB de Belo Horizonte promovendo saúde à população por meio do trabalho multiprofissional. Conhecer o perfil e atuação desse profissional no programa é fundamental para o planejamento das ações de saúde. Objetivo: Caracterizar as principais atividades realizadas e desafios encontrados durante o trabalho do fisioterapeuta no NASF-AB. Métodos: Estudo observacional de caráter transversal cuja amostra foi composta por 46 fisioterapeutas vinculados aos núcleos de Belo Horizonte que participaram da pesquisa por meio de um questionário eletrônico sobre o perfil profissional e a atuação no programa. Resultados e discussão: Após análise dos dados, identificou-se o atendimento individual como principal atividade realizada pelo fisioterapeuta apesar da maioria participar de quatro ou mais grupos de promoção à saúde. Para os profissionais a dimensão assistencial é a que mais contribui para o trabalho das eSF. Os desafios para assistência à população e realização do matriciamento foram falta de infraestrutura adequada e materiais, carga horária insuficiente e discussões pouco qualificadas. Uma das formas de superar algumas dessas dificuldades é organizar as ações no serviço e estimular o aperfeiçoamento dos profissionais mediante educação continuada e permanente. Conclusão: Os fisioterapeutas participantes atuam nas dimensões assistencial e técnico-pedagógica, com maior dificuldade nesta última. Entre os desafios destacam-se aspectos do apoio matricial, infraestrutura e demanda elevada de trabalho. Os resultados evidenciaram diferenças nos processos de trabalho do fisioterapeuta, reforçando que a complexidade e heterogeneidade dos territórios de saúde impõe diferentes desafios na atuação do profissional.
\end{abstract}

Palavras-chave: atenção primária à saúde; fisioterapia; sistema único de saúde.

\begin{abstract}
Background: Physiotherapists are present in all CFH-PC in Belo Horizonte, promoting health to the population through multidisciplinary work. Knowing the profile and role of this professional in the program is essential for planning health actions. Objective: To characterize the main activities performed and challenges encountered during the work of physical therapists at CFH-PC. Methods: Cross-sectional observational study whose sample consisted of 46 physical therapists linked to the centers of Belo Horizonte who participated in the survey through an electronic questionnaire on the professional profile and role in the program. Results and discussion: After analyzing the data, individual care was identified as the main activity performed by the physiotherapist, despite the majority participating in four or more health promotion groups. For professionals, the assistance dimension is the one that most contributes to the work of the tFH. The challenges for assisting the population and carrying out matrix
\end{abstract}

\footnotetext{
${ }^{1}$ Pontifícia Universidade Católica de Minas Gerais, Campus Betim, Brasil. E-mail: sabrinaviana@yahoo.com.br

${ }^{2}$ Pontifícia Universidade Católica de Minas Gerais, Campus Betim, Brasil. E-mail: dayaneribeiros@outlook.com

${ }^{3}$ Pontifícia Universidade Católica de Minas Gerais, Campus Betim, Brasil. E-mail: mclarabotelho7@gmail.com
} 
support were lack of adequate infrastructure and materials, insufficient workload and poorly qualified discussions. One of the ways to overcome some of these difficulties is to organize actions in the service and encourage the improvement of professionals through continuing and permanent education. Conclusion: Participating physiotherapists work in the care and technical-pedagogical dimensions, with greater difficulty in the latter. Among the challenges, aspects of matrix support, infrastructure and high labor demand stand out. The results showed differences in the physical therapists' work processes, reinforcing that the complexity and heterogeneity of health territories pose different challenges to their role.

Keywords: primary health care; physiotherapy; unified health system.

\section{Introdução}

O Núcleo Ampliado de Saúde da Família e Atenção Básica (NASF-AB), anteriormente nomeado Núcleo de Apoio à Saúde da Família (NASF), foi criado em 24 de janeiro de 2008 para apoiar a Estratégia de Saúde da Família (ESF) e ampliar as ações da Atenção Básica (AB) no cenário brasileiro $^{1,2}$. O objetivo desse programa é promover atendimento multiprofissional conforme a prioridade e a necessidade de saúde da população de determinada região.

Decorridos 13 anos desde a sua criação, pode-se dizer que o NASF-AB ainda é uma proposta inovadora $\mathrm{e}$ necessária para a $\mathrm{AB}$. Em Minas Gerais a implementação do programa ocorreu em períodos diferentes nos municípios. Em Belo Horizonte, os Núcleos foram implantados em 2008, mesmo ano de publicação da portaria ministerial $\mathrm{n}^{\mathrm{o}} 154^{1} \mathrm{e}$ várias ampliações ocorreram desde então. Atualmente, esse município possui 82 equipes de NASF-AB compostas por profissionais de saúde com formação distinta dos que integram a equipe de saúde da família (eSF). A constituição desse programa varia conforme o perfil epidemiológico da população e as prioridades do município, podendo incluir assistente social, educador físico, farmacêutico, fisioterapeuta, fonoaudiólogo, nutricionista, psicólogo e terapeuta ocupacional, dentre outros ${ }^{2,3}$. O papel desses profissionais é atuar em consonância com profissionais da eSF e contribuir para a integralidade do cuidado à saúde por meio da atuação clínica e ações de educação para a população adscrita ${ }^{4}$.

Entre os desafios impostos à atuação plena e eficaz dos profissionais do NASF$\mathrm{AB}$, estudiosos apontam dificuldades para efetivar o apoio matricial ${ }^{4,5}$ e compreender a dinâmica de trabalho ${ }^{6}$, somado à necessidade de administrar a carga horária para responder à alta demanda de atendimentos ${ }^{5,7}$.

$\mathrm{O}$ fisioterapeuta, em conjunto com os demais profissionais do NASF-AB, desempenha importante papel na $A B$, fundamentada na integralidade, compreensão territorial, humanização, interdisciplinaridade e intersetorialidade ${ }^{6}$. Assim, realiza ações de promoção da saúde, reabilitação e prevenção de doenças por meio de atendimentos individuais, coletivos e domiciliares dentro ou fora da Unidade Básica de Saúde (UBS $)^{8}$. A atuação do fisioterapeuta no NASF-AB desperta o interesse de pesquisadores, especialmente pelo caráter reabilitador que originou a profissão, deixando por muito tempo esse profissional excluído da AB. A atual proposta de formação profissional do fisioterapeuta é mais ampla e deve preparálo para atuar como generalista, assumindo responsabilidade pelas ações preventivas e de promoção à saúde, além do diagnóstico, tratamento e reabilitação em todos os ciclos de vida ${ }^{5}$.

Diante do exposto, este estudo objetivou caracterizar a atuação dos fisioterapeutas vinculados aos NASF-AB do município de Belo Horizonte, identificando as principais atividades realizadas e dificuldades encontradas durante o trabalho na $\mathrm{AB}$.

\section{Materiais e Métodos \\ Amostra e tipo de estudo}

Esta pesquisa foi um estudo observacional de corte transversal aprovada pelo Comitê de Ética em Pesquisa com os números
13319719.4.3001.5140 
13319719.4.0000.5137, cuja amostra de conveniência foi composta por todos os fisioterapeutas do NASF-AB do município de Belo Horizonte, totalizando 82 profissionais.

\section{Delineamento da pesquisa}

Os dados foram coletados no período de outubro de 2019 a março de 2020 por meio de um questionário eletrônico semiestruturado, constituído por 33 itens relacionados ao perfil e à atuação do fisioterapeuta no NASF-AB. O questionário foi elaborado pelos próprios pesquisadores e continha duas partes. A primeira, parte $\mathrm{A}$, abordou as características sociodemográficas e ocupacionais dos fisioterapeutas em 12 questões de múltipla escolha, enquanto a parte B foi composta por 22 perguntas sobre a atuação do profissional no NASF-AB, das quais quatro eram discursivas.

Os questionários autoaplicáveis foram encaminhados por via eletrônica aos profissionais, mediante anuência da secretaria de saúde competente. Após sete dias do envio do formulário, foram encaminhados lembretes por e-mail, com intervalo de sete dias entre eles.

\section{Critérios de Inclusão e Exclusão}

Foram incluídos os fisioterapeutas ativos na função durante o período de coleta dos dados (outubro de 2019 a março de 2020), que concordaram em participar da pesquisa.

\section{Procedimentos e Análise Estatística}

Posteriormente à obtenção dos questionários preenchidos, os dados foram informatizados e analisados por meio do programa estatístico SPSS (Statistical Package for Social Science, versão 22.0). Análise descritiva com medidas de tendência central (média, mediana), dispersão (desvio padrão), frequência e porcentagem foi conduzida para caracterizar a amostra e teste $T$ Student para análise bivariada (considerado alfa igual 0,05). Questões discursivas foram analisadas mediante análise temática, seguindo os passos: leitura e familiarização com os dados para identificar possíveis temas e padrões de ocorrência; redução dos dados e criação de categorias para análise; busca de temas que conferem significado; revisão e refinamento dos temas e análise detalhada a partir de pressupostos teóricos ${ }^{9}$.

\section{Resultados}

Ao todo foram enviados 82 questionários aos fisioterapeutas, dos quais 46 foram respondidos, o que representou uma taxa de resposta de 56,0\%. A amostra final do estudo incluiu 46 profissionais atuantes no NASF-AB de Belo Horizonte.

Em relação ao perfil sociodemográfico e ocupacional, houve predomínio do sexo feminino $(91,0 \%)$ e a idade variou de 26 a 58 anos, com valor médio igual a 37,7 anos ( $\mathrm{DP}= \pm 5,9)$. Quanto ao estado civil, $67,0 \%$ dos fisioterapeutas declararam estar casados ou em união estável. Os participantes tinham entre 3 e 29 anos de formado e 59,0\% deles cursaram a graduação em instituição privada. Sobre a titulação acadêmica, $\quad 72,0 \%$ dos profissionais possuíam especialização e ou residência em fisioterapia, $15,0 \%$ pósgraduação stricto sensu (mestrado ou doutorado) e 13,0\% apenas a graduação. O tempo médio de atuação no NASF-AB foi de 6,7 anos $(\mathrm{DP}= \pm 4,0)$, com valores: mínimo inferior a 1 ano e máximo de 12 anos. Houve predomínio da jornada de 20 horas semanais $(74,0 \%$ dos casos $)$ e aproximadamente metade dos profissionais atuavam em outro local como fisioterapeuta. A renda líquida mensal variou conforme o grau de titulação, atingindo valores maiores para profissionais com pós-graduação stricto sensu. Os resultados revelaram que os fisioterapeutas com mestrado ou doutorado que trabalham no NASF-AB em Belo Horizonte possuem, em média, uma renda líquida mensal 31,0\% superior comparada aos demais $(\mathrm{p}=0,023)$. 
Sobre a constituição dos NASF-AB, a análise dos dados evidenciou que, além do fisioterapeuta, as categorias mais representadas são: farmacêutico $(100,0 \%)$, nutricionista $(96,0 \%)$ e fonoaudiólogo (91,0\%). Ao serem questionados sobre a profissão que mais faz falta no NASF-AB, os fisioterapeutas indicaram o terapeuta ocupacional. A composição dos NASF-AB em relação à categoria profissional pode ser visualizada no Gráfico 1. É válido ressaltar que essa distribuição considerou os participantes da pesquisa e pode não representar a totalidade dos NASF-AB no município.

\section{Gráfico 1 - Composição dos NASF-AB em relação à categoria profissional}

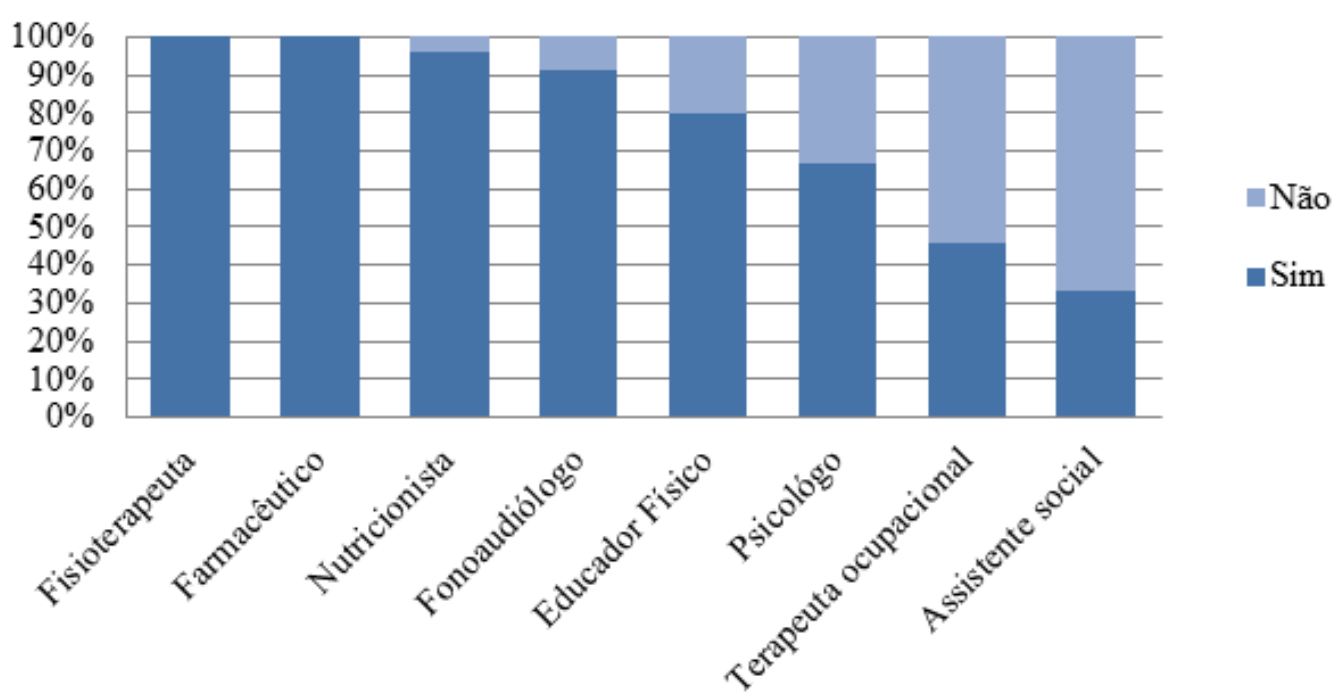

Fonte: elaborado pelas autoras

Considerando os aspectos relacionados ao trabalho, quase $60,0 \%$ das equipes de NASF-AB atuam em duas UBS e apoiam de cinco a dez eSF (Média $=8 ; \mathrm{DP}= \pm 2,2$ ). $\mathrm{Em}$ $54,0 \%$ dos Núcleos a carga horária total de fisioterapia soma $40 \mathrm{~h}$ semanais, o que indica a importância da categoria frente às necessidades de saúde da população.
Geralmente é prevista uma reunião de matriciamento mensal com o NASF-AB para cada eSF apoiada e os fisioterapeutas participam em média de $65,0 \%$ das reuniões, o que equivale a 4,6 reuniões por mês. As informações relativas à organização dos NASF-AB foram compiladas na Tabela 1.

Tabela 1 - Características do trabalho dos Fisioterapeutas no NASF-AB.

Tabela 1 - Características do trabalho dos Fisioterapeutas no NASF-AB.

\begin{tabular}{llll}
\hline Variável & Valor mínimo & Valor máximo & Média (DP) \\
$\begin{array}{l}\text { Carga horária semanal total dos profissionais do NASF- } \\
\text { AB }\end{array}$ & 120 & 280 & $174(47,8)$ \\
Número de eSF apoiadas & 5 & 10 & $7,6(1,3)$ \\
Reuniões de matriciamento previstas por mês & 3 & 10 & $7(1,9)$ \\
Número de Reuniões com participação do fisioterapeuta & 1 & 10 & $4,6(2,8)$ \\
\hline
\end{tabular}


Fonte: elaborada pelas autoras.

Os profissionais foram questionados sobre a atividade assistencial mais comum na prática clínica e o atendimento individual foi a modalidade mais citada $(59,0 \%$ dos casos). $\mathrm{O}$ atendimento compartilhado faz parte do cotidiano de trabalho do fisioterapeuta, ocorrendo pelo menos uma vez por mês (para $72,0 \%$ da amostra). O atendimento é mais comumente compartilhado com profissionais do próprio NASF-AB (em todos os casos), e em 52,0\% dos relatos, com profissionais da eSF. $\mathrm{Na}$ percepção dos entrevistados $(87,0 \%)$, os profissionais da eSF reconhecem o papel da fisioterapia na $\mathrm{AB}$.

Embora os atendimentos individuais ocupem consideravelmente a agenda, os fisioterapeutas atuam também em vários grupos. A maioria dos profissionais $(63,0 \%)$ coordena ou participa de quatro ou mais grupos de promoção à saúde. A maior parte dos fisioterapeutas $(65,0 \%)$ afirmou ter espaço para desenvolver ações coletivas e quase $90,0 \%$ declarou sentir-se apto para atuar em consonância com os princípios e diretrizes do SUS.

Ao questionar os profissionais sobre qual dimensão o NASF-AB mais contribui para o trabalho das eSF, $91,0 \%$ indicaram a assistencial (atendimento individual, coletivo e domiciliar) enquanto os demais citaram a dimensão técnico pedagógica (reuniões de matriciamento). Todos os participantes consideraram que a atuação do NASF-AB potencializa o trabalho das eSF. O Gráfico 2 representa a opinião dos fisioterapeutas sobre os elementos facilitadores da atuação no NASF-AB. Foi destacado o relacionamento com profissionais da própria equipe e da eSF.

\section{Gráfico 2 - Facilitadores da atuação no NASF-AB na percepção dos fisioterapeutas}

\section{Outros \\ Infraestrutura da UBS}

Educação continuada e cursos de aperfeiçoamento

Reconhecimento e valorização do trabalho pelo usuário

Apoio do gerente/ gestor

Relacionamentos com profissionais da eSF

Relacionamentos com profissionais do NASF- AB

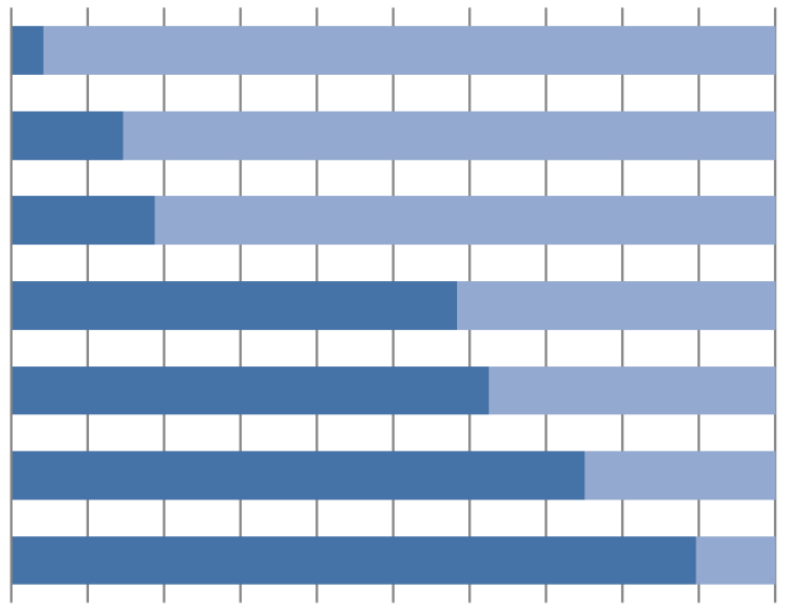

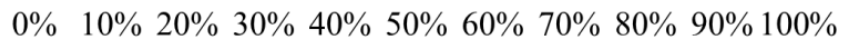

n Sim $\mathbf{n}_{\text {Não }}$

Legenda: UBS: Unidade Básica de Saúde, eSF: Equipe de Saúde da Família e NASF-AB: Núcleo Ampliado de Saúde da Família e Atenção Básica

Fonte: elaborado pelas autoras

Além das perguntas objetivas, o questionário eletrônico possuía também questões discursivas, dentre elas, três que abordaram as barreiras e facilitadores 
presentes na assistência à população e os desafios para desenvolver ações técnico pedagógicas. Análise qualitativa das respostas apontou para a existência de barreiras estruturais que prejudicam ações assistenciais, como falta de materiais e infraestrutura inadequada. Como exemplo foram citadas ausência de salas ou consultórios para atendimento individual e espaço físico inadequado para desenvolver atividades em grupo: "Falta de estrutura fisica da ubs" (entrevistado $n^{\circ} 32$ ); "Falta de espaço físico para atendimento" (consultórios ou espaços para práticas coletivas) (entrevistado $\left.n^{\circ} 02\right)$ e "falta de recursos materiais" (entrevistado $n^{\circ} 35$ ).

Outro desafio refere-se ao dimensionamento e carga horária das equipes de NASF-AB frente às demandas das eSF. De acordo com o entrevistado $\mathrm{n}^{\circ}$ 33, há uma "demanda mais elevada que a capacidade de atendê-la (horas semanais insuficientes)". Além da carga horária, foram citadas dificuldades para participar das reuniões de matriciamento e para trabalhar em equipe conforme demonstrado na seguinte fala: "dificuldade da equipe NASF trabalhar com eSF e até mesmo com os próprios profissionais do NASF na atuação de promoção e prevenção". (entrevistado $\left.n^{\circ} 14\right)$.

De acordo com os entrevistados, a influência do modelo biomédico na atuação dos profissionais da eSF e NASF-AB é uma barreira para o desenvolvimento das ações assistenciais à população: "atualmente $o$ trabalho está muito mais voltado para o curativo (atendimento individual) do que preventivo (grupos)", (entrevistado $n^{\circ} 12$ ). Ainda em relação à dimensão assistencial, foram apontados como empecilhos $\mathrm{o}$ deslocamento entre as unidades de saúde e a falta de apoio gerencial. Alguns profissionais elucidaram aspectos inerentes à população que prejudicam a assistência como: contexto social, crenças, nível de escolaridade, dificuldade de acesso aos serviços de saúde e absenteísmo nos atendimentos: "o pouco comprometimento da população assistida que falta muito aos atendimentos em grupo e individual", (entrevistado $n^{\circ} 18$ ).

Apesar de haver muitos desafios para a assistência na percepção dos fisioterapeutas, os resultados chamaram atenção, pois os mesmos aspectos apontados como barreiras, foram também valorizados e classificados como facilitadores por outros profissionais. $\mathrm{O}$ perfil profissional para atuar na atenção básica, apoio do gerente e das eSF, assim como a adesão da população facilitaram o processo de trabalho no NASF-AB de acordo com alguns fisioterapeutas, demonstrando que há diferenças na percepção e provavelmente no contexto de atuação dos núcleos. Somado a esses, foram destacados outros facilitadores como valorização do serviço pela população, espaço físico para o desenvolvimento das ações, autonomia profissional e reconhecimento do trabalho desenvolvido pelo NASF-AB.

Em relação à dimensão técnico pedagógica, parte da amostra $(\mathrm{N}=17)$ não reconhece desafios para realizar o apoio matricial, enquanto a maioria enfrenta dificuldades para efetivá-lo devido ao tempo insuficiente e incompatibilidade de carga horária, que por sua vez comprometem a construção do projeto terapêutico singular: "impossibilidade de participar de todas as reuniões de matriciamento devido a minha carga horária de 20 horas" (entrevistado $n^{\circ} 32$ ); "tempo insuficiente das eSF e da equipe do NASF para pensar planos terapêuticos singulares (nunca participei $d a$ elaboração) (entrevistado $\left.n^{\circ} 33\right)$ )". Outro desafio à realização do apoio matricial foi a limitação do próprio fisioterapeuta, que tem dificuldade de compreender o que é e como fazer o matriciamento na prática. Além disso, a falta de valorização pelas eSF que atribuem pouca importância a este apoio foi também considerado um desafio: "Muitas vezes as equipes querem apenas transferir o problema ou a demanda e não conhece o paciente" (entrevistado $\left.n^{\circ} 18\right) ;$ "há pouco interesse na discussão dos casos", 
(entrevistado $n^{\circ} 11$ ). A falta de vínculo entre usuários e eSF e a discussão pouco qualificada e aprofundada dos casos prejudica a qualidade das reuniões, dificultando também o apoio matricial.

\section{Discussão}

No Brasil, a implantação do NASF$\mathrm{AB}$ se deu em momentos distintos e a configuração de suas equipes não seguiu o mesmo padrão em todas as regiões, pois é influenciada pelo perfil epidemiológico da população e prioridades de cada município ${ }^{2,3}$. Em março de 2018 havia 5.221 núcleos ativos no país com grande atuação de fisioterapeutas, nutricionistas e psicólogos ${ }^{10}$. Em Belo Horizonte, a expressiva participação dos farmacêuticos no NASF-AB deve-se ao fato de que a Assistência Farmacêutica foi uma das 20 temáticas preconizadas no Plano Municipal de Saúde para o período 2018 a 2021. A meta para 2021 era garantir a presença de farmacêuticos (40h semanais) nas farmácias de todas as $\mathrm{UBS}^{11}$, e esses profissionais foram então vinculados às equipes de NASF-AB. Os profisssionais de Educação Física também foram vinculados aos NASF-AB, mas exercem suas atividades principalmente nas 79 academias da cidade, com foco nos grupos de atividade física. Psicólogos e assistentes sociais, com menor representatividade, são categorias profissionais que já existiam e atuavam na APS. Portanto, a implementação dos NASF-AB em Belo Horizonte trouxe um incremento dessas categorias, de forma que seu quantitativo no NASF-AB não corresponde à totalidade dos profissionais vinculados à APS.

Como preconizado nos documentos regulatórios do NASF-AB, faz parte do cotidiano dos fisioterapeutas a assistência individual, coletiva e domiciliar aos usuários. Neste e em outros estudos, os profissionais apontaram $\mathrm{o}$ atendimento individual como a atividade mais frequente $^{5,12}$. Fatores como sobrecarga de trabalho e falta de formação específica para o atendimento com grupos podem ocasionar a reprodução de práticas tradicionais e dificultar a organização e desenvolvimento de um grupo ${ }^{13}$.

As práticas coletivas são importantes meios de promoção à saúde e prevenção de agravos, constituindo ações que visam não somente atender às demandas, mas também fornecer espaços de socialização, apoio psicológico, aprendizado compartilhado e construção de planos em conjunto. Essas práticas objetivam tornar o sujeito autônomo e responsável por sua saúde e consequentemente, prevenir o adoecimento $^{2}$. As atividades em grupo corroboram para a acessibilidade à saúde pública, pois propiciam a participação popular embasada em suas especificidades e costumes ${ }^{14}$. O olhar positivo dos usuários sobre essas atividades expressam o impacto e capacidade dessas ações, que vão de encontro aos anseios e demandas dos participantes, sendo demonstradas pelo acolhimento, vínculo e cuidado ${ }^{14}$ que favorecem a construção do aprendizado e troca de vivências.

Deve-se considerar que a amostra entrevistada, apesar de sentir-se preparada para atuar na APS de acordo com os princípios do SUS, mencionou a demanda elevada das eSF como um fator de sobrecarga, além da falta de infra estrutura e de materiais. Em uma pesquisa de natureza qualitativa com profissionais da equipe de Saúde da Família do município de Ribeirão Preto, a falta de interesse do profissional, sobrecarga de trabalho, baixa adesão pelo usuário e limitações de espaço físico e materiais foram aspectos que dificultaram a realização das práticas grupais ${ }^{13}$. Por ser ferramenta essencial na promoção e educação em saúde, as diretrizes propostas para o NASF colocam os grupos como ações prioritárias na $\mathrm{AB}^{2}$. Dessa forma, a organização da agenda com horários compartilhados com a equipe e destinados ao planejamento de grupos surge como uma recomendação aos profissionais, aliada à educação continuada e 
aproximação de lideranças na comunidade para a identificação de equipamentos sociais que respondam às demandas locais de infraestrutura. Assim, é plausível pensar que o aumento da oferta de ações coletivas cause impacto positivo no processo de trabalho do fisioterapeuta, com redução das demandas de assistência individual.

A maioria dos fisioterapeutas afirmou que sua formação acadêmica foi realizada em consonância com as diretrizes do SUS, contribuindo para torná-los aptos a atuar na AB. As Diretrizes Curriculares Nacionais (DCN) do Curso de Graduação em Fisioterapia, importante marco para a transformação do ensino ${ }^{15}$, publicada em 2002, define uma formação generalista, humanística e crítica, de modo que permita o fisioterapeuta atuar em todos os níveis de atenção ${ }^{16}$. A formação acadêmica dos profissionais de saúde pautada nas competências exigidas pelo SUS é reafirmada nas DCN e cada Instituição de Ensino Superior (IES) possui a flexibilidade para elaborar e adaptar perfis de formação por meio das transformações e necessidades da sociedade ${ }^{17}$. Dessa forma, o ensino com enfoque no SUS não deve constar apenas em planejamentos, mas também na grade curricular e métodos de ensino ${ }^{17}$.

Diversas intervenções e estratégias foram realizadas para proporcionar mudanças nas graduações da área da saúde e aproximar os discentes do SUS, sendo elas o EducarSUS, AprenderSUS, VER-SUS, cursos de Formação de Facilitadores de Educação Permanente em Saúde e de Ativação de Mudanças ${ }^{18}$, Política Nacional de Educação Permanente em Saúde (PNEPS $)^{19}$, Pró-Saúde, PET Saúde e Residências profissionais ${ }^{18}$. Muitos avanços foram conquistados na formação do fisioterapeuta na direção de uma visão mais ampla de saúde, contudo, ainda há questões a serem aprimoradas e debatidas sobre a temática ${ }^{5,20,21}$.

Alguns participantes relataram influência do modelo biomédico na atuação de profissionais do NASF-AB e da eSF na assistência à população, com ênfase na doença, definida como um desarranjo ou falha de adaptação do corpo ao ambiente ${ }^{22}$. Com a reformulação da $\mathrm{AB}$, centrada na eSF, há um modelo de atuação que privilegia ações de promoção à saúde e prevenção de agravos, sendo centrado no indivíduo e nos coletivos ${ }^{23}$. Essa modalidade está embasada nos determinantes sociais do processo saúde doença $^{23}$. Dessa forma, entender a lógica biopsicossocial é fundamental para atuar na APS e não perpetuar ações biomédicas.

Neste estudo, os desafios para atuação do fisioterapeuta no NASF-AB foram abordados por dimensões de trabalho e seus resultados vão de encontro à maioria dos achados da literatura 4,5,6,7,8,20,13,24,25,26. Uma das formas de superar essas dificuldades é por meio do aperfeiçoamento dos profissionais mediante educação continuada que consiste na aquisição contínua de conhecimentos científicos e educação permanente que problematiza a atuação em saúde. Ambas favorecem aquisição de novos saberes e competências, proporcionando capacitação profissional, assistencial, renovação no processo e organização do trabalho ${ }^{27}$.

O principal desafio para a assistência à população foi a falta de infraestrutura e materiais que, em parte, justifica-se pelo fato das unidades de saúde não terem sido planejadas para receber equipes de NASF-AB. De acordo com as diretrizes do programa, os recursos destinados ao mesmo deverão suprir as demandas de espaço físico, transporte e materiais adequados para a realização das ações na UBS ou em locais comunitários ${ }^{2}$. Após a publicação da Nota Técnica 03$2020^{28}$, o município passou a ser o responsável pelo fornecimento desses recursos para o NASF-AB. Ressalta-se que o trabalho desenvolvido na $A B$ deve ser realizado priorizando a utilização de tecnologias leves como acolhimento, vínculo e escuta qualificada ${ }^{6,24}$. Mas, entende-se que a disponibilidade de espaços influencia positivamente a realização do serviço, assim como o vínculo com a 
comunidade e o apoio do gestor, que surgem como uma possibilidade de ampliação das oportunidades em relação ao espaço físico.

Outro ponto desafiador evidenciado neste estudo refere-se à qualidade das reuniões de matriciamento, fundamental para o apoio técnico pedagógico ${ }^{29}$. Fatores como despreparo do profissional, falta de valorização das reuniões pela eSF, fragilidade do vínculo entre eSF e usuários e o número de eSF apoiadas frente à carga horária de trabalho comprometem, na percepção dos físioterapeutas, a dimensão técnico pedagógica. Nos estudos de Formiga e Ribeiro ${ }^{5}$ e Braghini, Ferreti e Ferraz $^{7}$, a jornada de trabalho do fisioterapeuta também foi considerada insuficiente para atender as demandas de assistência à população e apoio matricial. Em relação à carga horária semanal no NASF-AB, há profissionais com 20 e 40 horas, mas o fisioterapeuta se enquadra apenas na primeira modalidade, pois, por lei, sua jornada de trabalho semanal deve ser de no máximo 30 horas $^{30}$. Para atender às necessidades do município, é comum a vinculação de dois fisioterapeutas com 20 horas semanais em um mesmo NASF-AB. Essa conformação ressalta a importância da comunicação entre os membros da equipe para que os casos sejam devidamente discutidos e compartilhados mesmo na ausência de algum integrante ${ }^{31,32}$.

Acredita-se que a criação recente do NASF-AB é outro desafio à efetivação do matriciamento conforme preconizado, acrescida das particularidades de cada território e da equipe. A qualidade do apoio matricial requer a interação entre NASF$\mathrm{AB}$ e eSF que, por sua vez, não pode ser determinada pela carga horária, e deve estar embasada em aspectos profissionais como multidisciplinaridade, vínculo, cuidado integral, acessibilidade, resolutividade e longitudinalidade ${ }^{33}$. As reuniões entre essas equipes ampliam o escopo das ações que serão desenvolvidas ${ }^{2,34}$, pois $\mathrm{o}$ matriciamento propicia e estimula um espaço para comunicação ativa e partilhamento de saberes entre a equipe de referência e a de apoiadores ${ }^{31}$. Por outro lado, sabe-se que a reunião não é o único espaço de troca e os profissionais de ambas as equipes podem aproveitar outras oportunidades de contato para interagir e compartilhar experiências. Portanto, o apoio matricial não deve ficar em segundo plano, e sim, mesclado à assistência, resultando em uma atuação pautada nas questões sociais e intrínsecas dos usuários ${ }^{2}$, capaz de aumentar a resolutividade dos problemas de saúde da população.

A organização das ações desenvolvidas no serviço ${ }^{34}$ e a qualificação dos profissionais mediante cursos de aperfeiçoamento $^{29}$, como exemplo, o curso oferecido pelo Ministério da Saúde para aperfeiçoar o apoio matricial na $\mathrm{AB}^{2}$, são mecanismos que qualificam as reuniões, assim como a contratação de novos profissionais a fim de aumentar a disponibilidade na agenda das equipes ${ }^{29}$. Outras formas de melhorar o apoio matricial ocorrem por meio do uso de instrumentos na interação com as equipes e usuários, garantia de carga horária para sua realização ${ }^{35}$, flexibilização na elaboração da agenda dos profissionais (MELO e col.2018), avaliação das práticas e resultados obtidos ${ }^{35,29,}$ constância nas reuniões e espaços institucionalizados paras as mesmas ${ }^{29}$. A todos os fatores citados, acrescenta-se a necessidade dos profissionais terem um olhar abrangente sobre o processo saúde-doença, embasado no modelo biopsicossocial ${ }^{36}$. Em um estudo sobre os desafios do NASF-AB em seus 10 anos de existência, cita-se estratégias como a ampliação da educação permanente conforme a configuração de cada equipe e território inserido, discussão da temática NASF e apoio matricial nos programas de residência, elaboração de ferramentas de avaliação e pesquisas que evidenciem os resultados do NASF-AB para consolidação do apoio matricial e do Núcleo $^{10}$.

O território é considerado uma área geográfica singular de elaboração 
descentralizada, onde são realizadas intervenções de promoção à saúde, prevenção de agravos, vigilância e recuperação da saúde ${ }^{37}$. Os territórios possuem formas específicas de adoecimento, mas, geralmente seus determinantes de saúde surgem de um âmbito geral $^{38}$. Dessa forma, é preciso considerar os diferentes contextos e singularidades de cada região, compreendendo seu dinamismo e não restringindo-se apenas à delimitação geográfica $^{39}$. Na mesma perspectiva, devese considerar as diferenças na atuação entre os profissionais do NASF-AB conforme as competências e particularidades individuais $^{2}$. Ao analisar as distintas percepções relatadas pelos fisioterapeutas sobre as barreiras e facilitadores presentes em sua atuação no cenário pesquisado, ficou evidente que a heterogeneidade nas respostas está relacionada às particularidades de cada regional pesquisada, características das eSF e dos NASF-AB, além de perfis distintos de profissionais Diante do exposto, é importante ressaltar que mudanças recentes na regulamentação do NASF-AB podem comprometer a continuidade do programa. Em novembro de 2019, a publicação da Portaria $\mathrm{n}^{\circ} 2.979^{40}$, e posteriormente da Nota Técnica $n^{\circ}$ 3/2020-DESF/SAPS/MS ${ }^{28}$, desvinculou normativas referentes ao funcionamento do NASF-AB e um novo cenário passou a vigorar. Uma das mudanças diz respeito à composição das equipes, que não estão mais relacionadas à tipologia do programa, sendo o gestor municipal responsável por definir seu funcionamento: carga horária, composição de equipes (NASF-eSF), financiamento e métodos. Compreende-se que o modo com que os gestores fazem a estruturação e acordos é fator condicionante para atuação do Núcleo ${ }^{10}$. Dessa forma, essa Nota Técnica $^{28}$ impôs um desafio novo à permanência dos NASF-AB e à consequente atuação do fisioterapeuta e de todos os demais profissionais que integram o programa.
Este foi o primeiro estudo que investigou a atuação e os desafios enfrentados pelo fisioterapeuta no NASF$\mathrm{AB}$ de Belo Horizonte e apresenta como limitações a representatividade amostral, visto que, em seis das nove regionais de saúde do município, obteve-se $40 \%$ ou menos de participação dos fisioterapeutas, o que pode influenciar os resultados encontrados, pois, as potencialidades e entraves de cada território são distintas. Outro ponto refere-se ao tamanho do questionário eletrônico, aparentemente extenso por possuir 34 perguntas, o que pode ter contribuído para respostas mais objetivas e menos aprofundadas nas questões discursivas.

\section{Conclusão}

$O$ Fisioterapeuta está inserido na $\mathrm{AB}$ por meio do NASF-AB e no município de Belo Horizonte a categoria tem atuado nas dimensões assistencial e técnicopedagógica, com maior dificuldade nesta última. Apesar de realizar atendimentos domiciliares e coletivos por meio de grupos, a atividade predominante na prática são os atendimentos individuais, frequentemente compartilhados com as demais categorias do NASF-AB. Na percepção dos fisioterapeutas, há dificuldade na atuação multiprofissional, realização do matriciamento em si e articulação entre demanda de trabalho e carga horária. Além disso, embora menos citadas, outras barreiras foram a prevalência do modelo biomédico na forma de atuação profissional e escassez de recursos materiais e infraestrutura.

Como facilitadores do trabalho, foram destacados o apoio do gestor e das eSF, perfil e autonomia profissional, disponibilidade de espaço físico e valorização do trabalho pela população. Os resultados evidenciaram uma heterogeneidade nas respostas relativas aos facilitadores e barreiras existentes na atuação do fisioterapeuta, demonstrando a complexidade e particularidade dos territórios de saúde e os distintos perfis 
profissionais. Conhecer os desafios presentes na prática do fisioterapeuta no NASF-AB pode contribuir para melhorias em sua atuação e dos demais profissionais, além de colaborar para processos de qualificação permanente. Por fim, após mudanças recentes no funcionamento do programa, é preciso compreender que as barreiras para atuação do fisioterapeuta ultrapassam aspectos intrínsecos ao trabalho no NASF-AB e relacionam-se também com decisões dos três níveis de governo.

\section{Referências Bibliográficas}

1. Ministério da Saúde (BR). Portaria n $n^{\circ} 154$, de 24 de janeiro de 2008. Cria os Núcleos de Apoio à Saúde da Família - NASF. Diário Oficial da União. 04 de março de 2008: 43.

2. Ministério da Saúde (BR). Núcleo de Apoio à Saúde da Família - Volume : Ferramentas para a gestão e para o trabalho cotidiano. Brasília; 2014.

3. Carvalho MS, et al. Necessidade e dinâmica da força de trabalho na Atenção Básica de Saúde no Brasil. Ciên. Saúde Colet. 2018; 23(1): 295-302.

4. Braghini CC, Ferretti F, Ferraz L. Atuação do fisioterapeuta no NASF: percepção dos coordenadores e da equipe. Fisioter. Mov. (Online). 2016; 29(4): 767-76.

5. Formiga NFB, Ribeiro KSQS. Inserção do Fisioterapeuta na Atenção Básica: uma Analogia entre Experiências Acadêmicas e a Proposta dos Núcleos de Apoio à Saúde da Família (NASF). Rev. bras. ciên. saúde. 2012; 16(2): 113-22.

6. Souza MC, Bonfim AS, Souza JN, Vilela ABA, Franco TB. Fisioterapia e núcleo de apoio à saúde da família: conhecimento, ferramentas e desafios. Mundo saúde (Impr.). 2013; 27(2): 176-84.

7. Braghini CC, Ferretti F, Ferraz L. Atuação do fisioterapeuta no contexto dos núcleos de apoio a saúde da família. Fisioter. Mov. (Online). 2017; 30(4): 703-13.

8. Barbosa EG, Ferreira DLS, Furbino SAR, Ribeiro EEN. Experiência da fisioterapia no Núcleo de Apoio à Saúde da Família em Governador Valadares, MG. Fisioter. Mov. (Online). 2010; 23(2): 323-30.

9. Minayo, MCS. Análise qualitativa: teoria, passos e fidedignidade. Ciên. Saúde Colet, 2012, 17(3), 621- 6 .

10. Melo EA, Miranda L, Silva AM, Limeira RMN. Des anos dos Núcleos de Apoio à Saúde da Família (NASF): problematizando alguns desafios. Saúde Debate. 2018; 42(1): 328-40.

11. SECRETARIA MUNICIPAL DE SAÚDE DE BELO HORIZONTE. Plano municipal de saúde 2018-2021. Belo Horizonte, 2018.

12. Fernandes JM, Rios TA, Sanches VS, Santos MLM. As ferramentas do NASF nas práticas em saúde de fisioterapeutas. Fisioter. Mov (Online), 29(4): 741-50, Oct./Dec. 2016.

13. Sanchez P. Práticas grupais e promoção da saúde: estratégia, contribuições e desafios. [Dissertação], Ribeirão Preto: Universidade de São Paulo, 1-73, 2018.

14. Fernandes ETP, Souza MNL,Rodrigues SM. Práticas de grupo do Núcleo de Apoio à Saúde da Família: perspectiva do usuário. Physis (impresso). 2019; 29(1):

15. 2-18.

16. Bispo Júnior JP. Formação em fisioterapia no Brasil: reflexões sobre a expansão do ensino e os modelos de formação. Hist. cienc. saude-Manguinhos [Internet]. 2009:16(3): 655-668.

17. CONSELHO NACIONAL DE EDUCAÇÃO CÂMARA DE EDUCAÇÃO SUPERIOR. Resolução CNE/CES. 4, de 19 de fevereiro de 2002. Institui Diretrizes 
Curriculares Nacionais do Curso de Graduação em Fisioterapia. Diário Oficial da União. 2002: Seção 1, p. 11.

18. Damiance RM, Panes VBC, Caldana ML, Bastos JRM. Formação acadêmica para o SUS $\mathrm{X}$ Competência pedagógica do formador: algumas considerações para o debate. Rev. Salusvita (Online). 2016: 35(3): 453-474.

19. Batista CB. Movimentos de reorientação da formação em saúde e as iniciativas ministeriais para as universidades. Barbarói. 2013; (38): 97-125.

20. Ministério da Saúde (BR). Secretaria de Gestão do Trabalho e da Educação na Saúde. Departamento de Gestão da Educação na Saúde. Política Nacional de Educação Permanente em Saúde: o que se tem produzido para o seu fortalecimento?. Brasília: Ministério da Saúde, 2018.

21. Souza MO, Santos KOB. Atuação do fisioterapeuta no Núcleo de Apoio à Saúde da Família. Fisioter. Mov. (Online). 2017; 30(2): 237-46.

22. Bim CR, Diamante C. Formação em fisioterapia: como a saúde coletiva está inserida nas instituições públicas de ensino superior do Paraná. Cad. Edu Saúde e Fis. 2020; 7(13): 213.

23. Puttini RF, Junior AP, Oliveira LR. Modelos explicativos em saúde coletiva: abordagem biopsicossocial e auto-organização. Physis. 2010; 20(3): 753-67.

24. Esmeraldo GROV, Oliveira LC, Filho CEE, Queiroz DM. Tensão entre o modelo biomédico e a Estratégia de Saúde da Família: a visão dos trabalhadores de saúde. Rev. APS. 2017; 20(1): 98-106.

25. Souza MC, Bonfim AS, Souza JN, Vilela ABA, Franco TB. Fisioterapia e núcleo de apoio à saúde da família: um estudo sob a ótica dos gestores, profissionais e usuários de saúde da família. Rev. APS. 2014; 17(2): 189-94.

26. FILHO AVD, Vieira, Aveiro MC. Atuação dos fisioterapeutas dos núcleos de apoio à saúde da família entre idosos do município de Arapiraca-Al, Brasil. Rev. bras. promoç. saúde (Impr.). 2012; 25(4): 397-404.

27. Belettini NP, et al. Fisioterapeutas integrantes do Núcleo de Apoio à Saúde da Família do Estado de Santa Catarina: competências e desafios. Fisioter. Bras.. 2013; 14(6): 4338 .

28. Silva ICB, Silva LAB, Valença AMG, Sampaio J. O processo de trabalho do núcleo ampliado de saúde da família e atenção básica. Trab. Educ. Saúde. 2019; 17(1): 1-19.

29. BRASIL. NOTA TÉCNICA No 3/2020-DESF/SAPS/MS, de 28 de janeiro de 2020. Dispõe sobre o Núcleo Ampliado de Saúde da Família e Atenção Básica (NASF-AB) e o Programa Previne Brasil. Ministério da Saúde, Secretaria de Atenção Primária à Saúde Departamento de Saúde da Família.

30. Treichel CAS, Campos RTO, Campos GWS. Impasses e desafios para consolidação e efetividade do apoio matricial em saúde mental no Brasil. Interface comun. saúde educ.. $2019 ; 23$.

31. Brasil. Lei $\mathrm{n}^{\circ}$ 2.436, de 01 de março de 1994. Fixa a Jornada de Trabalho dos Profissionais Fisioterapeutas e Terapeuta Ocupacional. Brasília 1 de març 1994.

32. Campos GWS, Domitti AC. Apoio matricial e equipe de referência: uma metodologia para gestão do trabalho interdisciplinar em saúde. Cad. Saúde Pública. 2007; 23(2): 399-407.

33. Ministério da Saúde (BR), Secretaria de Atenção à Saúde, Departamento de Atenção Básica. Práticas em reabilitação na AB: o olhar para a funcionalidade na interação com o território. Ministério da Saúde: Brasília, 2017.

34. Hirdes A. A perspectiva dos profissionais da Atenção Primária à Saúde sobre o apoio matricial em saúde mental. Ciên. Saúde Colet. 2015; 20(2).

35. Pannizi M, Lacerda JT, Natal S, Franco TB. Reestruturação produtiva na saúde: atuação e desafios do Núcleo de Apoio à Saúde da Família. Saúde Debate. 2017; 41(112): 155-70. 
36. Castro CP, Oliveira MM, Campos GWS. Apoio Matricial no SUS Campinas: análise da consolidação de uma prática interprofissional na rede de saúde. Ciên. Saúde Colet. (Impresso). 2016; 21(5): 1625-36.

37. Bezerra E, Dimenstein, M. Os CAPS e o trabalho em rede: tecendo o apoio matricial na atenção básica. Psicol. ciênc. prof. 2008; 28(3): 632-645.

38. Ministério da Saúde (BR). Portaria $\mathrm{n}^{\circ}$ 2.979, de 12 de novembro de 2019. Institui o Programa Previne Brasil, que estabelece novo modelo de financiamento de custeio da Atenção Primária à Saúde no âmbito do Sistema Único de Saúde, por meio da alteração da Portaria de Consolidação $n^{0}$ 6/GM/MS, de 28 de setembro de 2017. Diário Oficial da União. 13 nov 2019: 220.

39. Santos AL, RIGOTTO, RM. Território e territorialização: incorporando as relações produção, trabalho, ambiente e saúde na atenção básica à saúde. Trab. educ. saúde (Online). 2010; 8(3): 387-406.

40. Tetemann EC, Trugilho SM, Sogame LCM. Universalidade e Territorialização no SUS: contradições e tensões inerentes. Textos contextos (Porto Alegre). 2016; 15(2): 356-69.

41. Ministério da Saúde (BR). Portaria $\mathrm{n}^{\circ}$ 2.979, de 12 de novembro de 2019. Institui o Programa Previne Brasil, que estabelece novo modelo de financiamento de custeio da Atenção Primária à Saúde no âmbito do Sistema Único de Saúde, por meio da alteração da Portaria de Consolidação $n^{0}$ 6/GM/MS, de 28 de setembro de 2017. Diário Oficial da União. 13 nov 2019.

\section{Como citar este artigo:}

Viana SO, Silva DJR, Amorim MCBV. Atuação do fisioterapeuta no NASF-AB: possibilidades e desafios. Rev. Aten. Saúde. 2021; 19(70): 277-289. 